\title{
Participação no programa de atividade física de idosos residentes em uma Casa Gerontológica no Rio de Janeiro
}

\author{
Participation in a physical activity program amongst \\ elderly residents of a geriatric home in Rio de Janeiro
}

\author{
Simone Mattos do Nascimento ${ }^{1}$, Rejane Sobrino Pinheiro ${ }^{2}$
}

\begin{abstract}
Resumo
Objetivo: Analisar os fatores associados à participação dos residentes na Casa Gerontológica de Aeronáutica Brigadeiro Eduardo Gomes (CGABEG) no Programa de Caminhada e Atividade Física da instituição. Metodologia: Foi realizado um estudo transversal com idosos, que, no momento da admissão, receberam avaliação funcional baseada em instrumento desenvolvido pela instituição, abordando aspectos físicos, realização de atividades de vida diárias, instrumentais e sociais. Foi realizado levantamento de comorbidades em prontuário e os idosos foram entrevistados sobre escolaridade e história pregressa e atual da prática de exercício, além da autoavaliação do estado de saúde. Foi elaborado um modelo de regressão logística para analisar a associação entre a prática de exercício e as variáveis do estudo. Resultados: Dentre os 94 residentes selecionados, 53,2\% aderiram à prática de atividade física, quase exclusivamente (88\%). Um quarto dos idosos sedentários aderiu ao programa após admissão na CGABEG. Foram observadas diferenças, mas não estatisticamente significantes, apontando maior prática para os homens, os com menor grau de dependência, menos agravos e mais escolaridade. Observou-se diferença estatisticamente significante apenas para a autoavaliação do estado de saúde (maior prática para aqueles com melhor autoavaliação). Conclusão: A incorporação de programa de atividade física orientado ao idoso é uma estratégia que pode potencializar a adesão e a manutenção da prática de atividade física. Embora sem significância estatística, escolaridade não mostrou associação, diferente da literatura sobre o tema, provavelmente por se tratar de população idosa, cujos comportamentos dependentes do gênero são bastante diferenciados dos da população geral.
\end{abstract}

Palavras-chave: atividade física; idoso; estilo de vida sedentário.

\begin{abstract}
Objective: The purpose of this study was to analyze the factors related to the participation of the residents of the Air Force's geriatric home Casa Gerontológica de Aeronáutica Brigadeiro Eduardo Gomes (CGABEG) in the Walking and Physical Activity Program offered by the institution. Methods: A cross-sectional study was conducted with elderly people who lived in the geriatric home. It was performed a functional evaluation at the time of the admission, based on a tool developed by the institution, addressing physical aspects, activities of daily living, instrumental and social performance. The data of comorbidities were obtained from medical records. The elderly were interviewed on education, history and current practice of exercise, frequency, type and duration of physical activity practiced in addition to the self-assessment of health status. It was drawn a logistic regression model to analyze the association between the practice of physical activity and the variables of the study. Results: Among the 94 residents selected to join the study, 53.2\% were practicing some kind of activity and $88 \%$ of these were involved in walking. A quarter of sedentary elderly took part in the program after being admitted in the geriatric home. Differences were found, although they were not statistically significant. The results point out that the ones
\end{abstract}

Trabalho realizado no Instituto de Estudos em Saúde Coletiva da Universidade Federal do Rio de Janeiro (UFRJ) - Rio de Janeiro (RJ), Brasil.

${ }^{1}$ Fisioterapeuta; Especialista em Geriatria e Gerontologia pela Universidade Federal Fluminense (UFF) - Niterói (RJ), Brasil; Especialista em Fisioterapia em Oncologia pelo Instituto Nacional do Câncer (INCA) - Rio de Janeiro (RJ), Brasil.

2Doutora em Saúde Pública; Professora Adjunta do Departamento de Medicina Preventiva da Faculdade de Medicina e do Instituto de Estudos em Saúde Coletiva da UFRJ - Rio de Janeiro (RJ), Brasil.

Endereço para correspondência: Simone Mattos do Nascimento - Instituto de Estudos em Saúde Coletiva da UFRJ - Praça Jorge Machado Moreira, s/n - Cidade Universitária - CEP: 21944-970 - Rio de Janeiro (RJ), Brasil -E-mail: simonemtn@uol.com.br

Fonte de financiamento: nenhuma.

Conflito de interesse: nada a declarar. 
who practice more are men with less dependency, less injuries and more schooling. A statistically significant difference was found only for the auto-evaluation of the health state (more practice to the ones with best auto-evaluation). Conclusion: The incorporation of an elderly-oriented physical activity program is a strategy which can potentiate the participation and keep up the physical activity. Although not statistically significant, education showed no association, in contrast to the literature on the subject, probably because gender relations are different comparing elderly and the general population.

Keywords: physical activity; aged; sedentary lifestyle; health programs.

\section{INTRODUÇÃO}

A prática da atividade física sistemática exerce um importante papel na melhoria e manutenção do desempenho das atividades da vida diária do indivíduo, especificamente para a pessoa idosa, reduzindo o impacto do déficit funcional natural, causado pelo seu processo de envelhecimento ${ }^{1,2}$. O nível de atividade física é um componente importante na estabilização e na redução da progressão de várias doenças crônicas associadas ao aumento da idade ${ }^{3}$. Tem sido observada maior longevidade com o aumento da atividade física, redução no risco de doenças cardiovasculares, acidente vascular cerebral e câncer e diminuição de todas as causas de mortalidade ${ }^{4,5}$. Nesse sentido, a prática da atividade física por meio de programas direcionados à clientela idosa pode ser vista como um recurso promissor ${ }^{6}$.

O sedentarismo atinge parcelas elevadas da população, independentemente do critério utilizado para a definição de atividade física e da população estudada. A literatura nacional e internacional indica que o sedentarismo atinge aproximadamente entre 60 e $80 \%$ da população adulta ${ }^{7,8}$. A prática da atividade física reduz com o aumento da idade e é menor entre as mulheres que entre os homens ${ }^{8-10,12}$ e cresce com o aumento da escolaridade $\mathrm{e}^{1,11,14,16}$.

O problema do sedentarismo é resultado da baixa taxa de adesão inicial e da alta taxa de desistências, que é de aproximadamente $50 \%$ a taxa de desistência após os 6 meses iniciais $^{12}$. Segundo Ferreira et al. ${ }^{13}$, mudanças na atividade física de idosas fisicamente ativas podem ocorrer a partir de orientações que estimulem a prática de atividade física moderada, em especial a caminhada, embora não tenham analisado a permanência a longo prazo dessa mudança comportamental.

Assim, o objetivo deste estudo foi analisar os fatores associados à participação de idosos em um programa de atividade física, investigando os residentes de uma casa gerontológica.

\section{METODOLOGIA}

Foi realizado um estudo transversal com os idosos admitidos na Casa Gerontológica de Aeronáutica Brigadeiro Eduardo Gomes (CGABEG) - Ministério da Aeronáutica, na Ilha do Governador, Rio de Janeiro, no período de março de 1985 até dezembro de 1999. A instituição admitia apenas militares e seus dependentes e possuía um programa de exercício e caminhada, ministrado por fisioterapeutas. Os residentes na CGABEG eram constantemente convidados e estimulados à participação. A instituição conta com programas de terapia ocupacional, programação social, além de um hospital geriátrico e gerontológico e serviço ambulatorial para a população residente na área onde se localiza a CGABEG.

Foram analisados, inicialmente, 119 idosos. Todos eles foram submetidos a uma avaliação funcional na admissão na CGABEG. O serviço de admissão possuía equipe multidisciplinar composta de um médico, uma enfermeira, uma assistente social e um psicólogo, que avaliavam individualmente a condição física, mental e social do idoso. Os aspectos abordados na avaliação funcional foram (Quadro 1): psicológicos - a lucidez da consciência, a orientação espaço-temporal, a memória, o pensamento, a percepção e a capacidade de autodeterminação; físicos - a deambulação, o déficit visual, o controle de esfíncteres, a capacidade de ter iniciativa e desempenho nas suas atividades cotidianas e instrumentais (alimentação, higiene, locomoção, vestuário, adequação pessoal, telefonar, fazer compras, receber pensão, pegar condução, ir ao banco). Na admissão, a equipe classificava o idoso nas seguintes categorias: residente gerontológico independente (RGOI); residente gerontológico de leve dependência (RGOL); residente geriátrico de média dependência (RGEM) e residente geriátrico de total dependência (RGET).

Foram excluídos da análise 25 idosos: os classificados como RGET, os que estavam internados em ambiente hospitalar, os que estavam viajando, os que faleceram no período da pesquisa.

Os 94 idosos selecionados foram entrevistados sobre escolaridade, história pregressa e atual da prática de exercício; frequência semanal, modalidade e duração em minutos da atividade física praticada; e autoavaliação do estado de saúde. Esta foi medida mediante o relato subjetivo do idoso, o qual pontuou de 0 a 10 a sua saúde física. Foi coletada em prontuário informação relativa às comorbidades.

Foi calculado um índice de morbidade relacionado à presença de agravos à saúde para os quais o sedentarismo é considerado um fator de risco. Esse índice corresponde à contagem de doenças, apresentadas por cada idoso, que estivessem classificadas nas categorias: doenças cardiovasculares 
Quadro 1. Classificação funcional segundo a Casa Gerontológica de Aeronáutica Brigadeiro Eduardo Gomes

\begin{tabular}{|c|c|c|c|c|}
\hline \multicolumn{5}{|c|}{ Grau de classificação funcional } \\
\hline Item & RGOI & RGOL & RGEM & RGET \\
\hline Lucidez & Preservada & $\begin{array}{c}\text { Preservada/ levemente } \\
\text { alterada }\end{array}$ & Alterada episódica & Significativamente alterada \\
\hline Orientação espaço-temporal & Preservada & $\begin{array}{l}\text { Preservada/ levemente } \\
\text { alterada }\end{array}$ & $\begin{array}{c}\text { Preservada/ } \\
\text { significativamente alterada }\end{array}$ & Significativamente alterada \\
\hline Memória & Preservada & Levemente alterada & $\begin{array}{c}\text { Levemente/ } \\
\text { severamente } \\
\text { alterada }\end{array}$ & $\begin{array}{l}\text { Severamente } \\
\text { alterada }\end{array}$ \\
\hline Pensamento & Preservada & Preservado/ alterado & $\begin{array}{l}\text { Levemente/ } \\
\text { severamente } \\
\text { alterada }\end{array}$ & $\begin{array}{l}\text { Severamente } \\
\text { alterada }\end{array}$ \\
\hline Percepção & Preservada & Preservada & Alterada & Significativamente alterada \\
\hline $\begin{array}{l}\text { Capacidade de } \\
\text { autodeterminação }\end{array}$ & Preservada & Supervisão & $\begin{array}{l}\text { Supervisão/ } \\
\text { incapaz }\end{array}$ & Incapaz \\
\hline Deambulação & Preservada & Preservada/ auxílio eventual & $\begin{array}{l}\text { Auxílio permanente/ } \\
\text { acamado }\end{array}$ & Acamado \\
\hline $\begin{array}{l}\text { Déficit visual bilateral } \\
\text { significativo }\end{array}$ & Não apresenta & Não apresenta & Apresenta & Apresenta \\
\hline Esfíncteres & Controla & Controla/não controla & $\begin{array}{c}\text { Controla/não } \\
\text { controla }\end{array}$ & Não controla \\
\hline $\begin{array}{l}\text { Tem iniciativa e condições de } \\
\text { assumir responsabilidade }\end{array}$ & Sim & $\begin{array}{c}\text { Preservada/ } \\
\text { Auxílio ocasional }\end{array}$ & $\begin{array}{l}\text { Auxílio para as AVD e não } \\
\text { realiza as AIVD }\end{array}$ & $\begin{array}{c}\text { Não realiza as AVD } \\
\text { e as AIVD }\end{array}$ \\
\hline Cuidados da enfermagem & Não & Sim ocasional & Sim permanente & Sim permanente \\
\hline
\end{tabular}

RGOI: residente gerontológico independente; RGOL: residente gerontológico de leve dependência; RGEM: residente geriátrico de média dependência; RGET: residente geriátrico de total dependência; AVD: atividades da vida diária; AIVD: atividades instrumentais da vida diária.

(hipertensão, acidente vascular encefálico e isquemia aguda transitória); doenças musculoesqueléticas (tráumato-ortopédica e reumatológica); doenças endócrinas (diabete mellitus); doenças neuromusculares.

Foi realizada análise bivariada dos fatores estudados com o fato de o residente fazer ou não exercício, utilizando-se o teste $t$ para as variáveis contínuas e o $\chi^{2}$ para as categóricas, com nível de significância $\alpha=0,05$. Foi realizada regressão logística para analisar a associação entre a prática de exercício e as variáveis do estudo. Foi utilizado como critério de inclusão das variáveis no modelo aquelas que apresentassem significância estatística de até $\alpha=0,20$ na regressão simples e que permanecessem com significância $\alpha=0,05$ no modelo multivariado ${ }^{14}$. As análises foram realizadas com os programas Epi Info versão 6.04 e SPSS versão 10.0.

\section{RESULTADOS}

A maioria dos idosos analisados era do sexo feminino (79,8\%), com média de idade de 81,6 anos, com desvio padrão de 7,5 anos (Tabela 1). Observou-se certa uniformidade em relação à escolaridade. O grau de dependência dos idosos apresentou a seguinte distribuição: 39,4\% residente gerontológico independente; $35,1 \%$ residente gerontológico de leve dependência e 25,5\% geriátrico de média dependência. O tempo médio de permanência na instituição foi de 4,5 anos (desvio padrão de 3,7 anos) e a média da autoavaliação do seu estado de saúde foi 7,7 (desvio padrão 2,4).

À época da pesquisa, $53,2 \%$ dos idosos praticavam atividade física, $24,5 \%$ pararam e não retornaram e $22,3 \%$ nunca praticaram (Tabela 1). Pôde-se constatar que um número considerável de idosos (25,5\%), que nunca foram adeptos a uma prática regular de atividade física, aderiu ao programa de caminhada e exercício proposto pela instituição ou praticou atividade física fora do programa, após serem admitidos na CGABEG. Dos que praticavam atividade física, a maioria realizava caminhadas $(88 \%), 58 \%$ a faziam diariamente por 60 minutos ou mais e $20 \%$ por pelo menos 30 minutos, no mínimo 3 vezes por semana (dados não apresentados).

Constatou-se que um pouco menos de $15 \%$ dos idosos não apresentavam agravo de saúde para o qual o sedentarismo é fator de risco, $41,5 \%$ possuíam pelo menos um agravo, uma porcentagem um pouco menor possuía de $2(26,6 \%)$ a 3 $(13,8 \%)$ agravos e apenas $3,2 \%$ possuíam 4 agravos de saúde para os quais o sedentarismo é fator de risco para a manutenção e melhoria de sua condição de saúde e qualidade de vida. As doenças mais frequentes foram as cardiovasculares, 
Tabela 1. Caracterização dos idosos residentes na CGABEG (1999)

\begin{tabular}{|c|c|c|}
\hline Variáveis & $\mathbf{n}$ & $\%$ \\
\hline \multicolumn{3}{|l|}{ Sexo } \\
\hline Masculino & 19 & 20,2 \\
\hline Feminino & 75 & 79,8 \\
\hline \multicolumn{3}{|l|}{ Idade (anos) } \\
\hline 65 a 69 & 3 & 3,2 \\
\hline 70 a 74 & 19 & 20,2 \\
\hline 75 a 79 & 15 & 16,0 \\
\hline 80 a 84 & 20 & 21,3 \\
\hline 85 a 89 & 24 & 25,5 \\
\hline 90 a 94 & 8 & 8,5 \\
\hline 95 a 99 & 4 & 4,3 \\
\hline 100 a 104 & 1 & 1,0 \\
\hline Média \pm desvio padrão (anos) & $81,6 \pm 7,5$ & \\
\hline \multicolumn{3}{|l|}{ Escolaridade } \\
\hline $1^{\circ}$ grau incompleto & 25 & 26,6 \\
\hline $1^{\circ}$ grau completo & 24 & 25,5 \\
\hline $2^{\circ} \mathrm{grau}$ & 27 & 28,7 \\
\hline superior & 18 & 19,2 \\
\hline \multicolumn{3}{|l|}{ Grau de dependência } \\
\hline RGOI & 37 & 39,4 \\
\hline RGOL & 33 & 35,1 \\
\hline RGEM & 24 & 25,5 \\
\hline \multicolumn{3}{|l|}{ Tempo de permanência (anos) } \\
\hline Média \pm desvio padrão (anos) & $4,6 \pm 3,7$ & \\
\hline \multicolumn{3}{|c|}{ Autoavaliação do estado de saúde (0-10) } \\
\hline Média \pm desvio padrão (anos) & $7,7 \pm 2,4$ & \\
\hline \multicolumn{3}{|l|}{ Atividade física } \\
\hline Iniciou a prática na CGABEG & 24 & 25,5 \\
\hline Sempre fez & 18 & 19,2 \\
\hline Parou e retornou & 8 & 8,5 \\
\hline Parou e não retornou & 23 & 24,5 \\
\hline Nunca fez & 21 & 22,3 \\
\hline \multicolumn{3}{|l|}{ Número de Agravos } \\
\hline Nenhum & 14 & 14,9 \\
\hline Um agravo & 39 & 41,5 \\
\hline Dois agravos & 25 & 26,6 \\
\hline Três agravos & 13 & 13,8 \\
\hline Quatro agravos & 3 & 3,2 \\
\hline Total & 94 & 100,0 \\
\hline
\end{tabular}

RGOI: residente gerontológico residente; RGOL: residente gerontológico de leve dependência; RGEM: residente geriátrico de média dependência; CGABEG: Casa Gerontológica de Aeronáutica Brigadeiro Eduardo Gomes

com $37,2 \%$ de idosos apresentando hipertensão arterial sistêmica, $43,6 \%$ com outra doença do coração e $4,3 \%$ tendo sofrido acidente vascular encefálico (Tabela 2). Uma porcentagem significativa apresentou doenças musculoesqueléticas $(38,3 \%)$, seguidas de depressão $(19,1 \%)$ e doenças do trato gastrointestinal $(18,1 \%)$.

Foram observadas pequenas diferenças (Tabela 3), mas que não foram estatisticamente significantes, entre a prática de atividade física e: sexo (maior prática para os homens);
Tabela 2. Distribuição de morbidade dos idosos residentes na Casa Gerontológica de Aeronáutica Brigadeiro Eduardo Gomes (1999)

\begin{tabular}{lcc} 
Doenças & n & $\%$ \\
Hipertensão arterial sistêmica & 35 & 37,2 \\
Outras doenças do coração & 41 & 43,6 \\
Acidente vascular encefálico & 4 & 4,3 \\
Depressão & 18 & 19,1 \\
Doenças do trato gastrointestinal & 17 & 18,1 \\
Doenças musculoesqueléticas & 36 & 38,3 \\
Doenças neurológicas & 8 & 8,5 \\
Diabetes mellitus/Endócrinas & 16 & 17,0 \\
Ópticas & 16 & 17,0 \\
Tumores/Câncer & 6 & 6,4 \\
Geniturinária & 6 & 6,4 \\
Respiratórias & 2 & 2,1 \\
Outros & 6 & 6,4 \\
\hline
\end{tabular}

escolaridade (menor prática para aqueles com menos escolaridade); número de agravos para os quais o sedentarismo é fator de risco (menor prática para aqueles com mais de três agravos); grau de dependência (RGOI e RGOL praticam mais atividade física); e tempo de permanência na instituição (maior prática para os mais novos na Casa $-4,1$ versus 5,1 anos). Observou-se associação estatisticamente significante para a autoavaliação do estado de saúde (maior prática para aqueles com melhor autoavaliação, $p=0,03$ ).

No modelo de regressão simples, apenas a autoavaliação e possuir três ou mais agravos em relação a nenhum, um ou dois agravos mostraram significância na explicação das chances de um idoso realizar exercício físico. No entanto, no modelo final, a variável referente aos agravos foi eliminada por não apresentar significância estatística e não ter melhorado o ajuste do modelo. Assim, foi apresentada apenas a razão de chances bruta para cada variável. Para cada ponto a mais na autoavaliação do idoso, observou-se um aumento de $23 \%$ nas chances de realização de exercício.

\section{DISCUSSÃO}

No presente estudo, observou-se que $46,8 \%$ dos idosos praticavam atividade física à época da pesquisa. Esse percentual foi superior aos 30\% citados por Allison e Keler ${ }^{2}$ em estudos prévios sobre indivíduos acima de 65 anos, assim como foi superior ao percentual abaixo de $20 \%$ observado em estudo representativo da população idosa do município do Rio de Janeiro $^{14}$, e de menos de $10 \%$ em estudo com população idosa do Porto, Portugal'. Porém, foi próximo ao encontrado (42\%) em estudo de população acima de 65 anos residente em área de influência de unidades básicas de saúde e de unidades do Programa de Saúde da Família do Nordeste e do Sul do Brasil ${ }^{15}$. 
Tabela 3. Atividade física, segundo os fatores estudados

\begin{tabular}{|c|c|c|c|c|c|c|}
\hline \multirow{3}{*}{ Variáveis } & \multicolumn{4}{|c|}{ Atividade física } & \multirow{3}{*}{ RC } & \multirow{3}{*}{ IC95\% } \\
\hline & \multicolumn{2}{|c|}{$\operatorname{sim}$} & \multicolumn{2}{|c|}{ não } & & \\
\hline & $\mathrm{n}$ & $\%$ & $\mathrm{n}$ & $\%$ & & \\
\hline \multicolumn{7}{|l|}{ Sexo } \\
\hline Feminino & 38 & 50,7 & 37 & 49,3 & 1,00 & - \\
\hline Masculino & 12 & 63,2 & 7 & 36,8 & 1,67 & $0,59-4,71$ \\
\hline \multicolumn{7}{|l|}{ Grau de instrução } \\
\hline $1^{\circ}$ grau incompleto & 11 & 44,0 & 14 & 56,0 & 0,79 & $0,23-2,65$ \\
\hline $1^{\circ}$ grau completo & 15 & 62,5 & 9 & 37,5 & 1,67 & $0,48-5,76$ \\
\hline $2^{\circ}$ grau completo & 15 & 55,6 & 12 & 44,4 & 1,25 & $0,38-4,13$ \\
\hline Superior & 9 & 50,0 & 9 & 50,0 & 1,00 & - \\
\hline \multicolumn{7}{|l|}{ Morbidade } \\
\hline Nenhum agravo & 9 & 64,3 & 5 & 35,7 & 1,00 & - \\
\hline Um agravo & 21 & 53,8 & 18 & 46,2 & 0,65 & $0,18-2,29$ \\
\hline Dois agravos & 15 & 60,0 & 10 & 40,0 & 0,83 & $0,22-3,23$ \\
\hline Três ou quatro agravos & 5 & 31,3 & 11 & 68,7 & 0,25 & $0,06-1,16$ \\
\hline \multicolumn{7}{|l|}{ Grau de dependência } \\
\hline RGOI & 21 & 56,8 & 16 & 43,2 & 1,14 & $0,43-2,81$ \\
\hline RGOL & 18 & 54,5 & 15 & 45,5 & 1,00 & - \\
\hline RGEM & 11 & 45,8 & 13 & 54,2 & 0,71 & $0,25-2,03$ \\
\hline Tempo de permanência (anos) & & 4,1 & & 5,1 & 0,92 & $0,82-1,03$ \\
\hline Idade média (anos) & & 81,1 & & 82,3 & 0,98 & $0,98-1,03$ \\
\hline Autoavaliação* & & 8,2 & & 7,1 & 1,23 & $1,01-1,49$ \\
\hline Total & 50 & 53,2 & 44 & 46,8 & & \\
\hline
\end{tabular}

${ }^{*} \mathrm{p}<0,05$, teste $t$ para as variáveis contínuas e $\chi^{2}$ para as categóricas; RGOI: residente gerontológico residente; RGOL: residente gerontológico de leve dependência; RGEM: residente geriátrico de média dependência; RC: Razão de Chance; IC: intervalo de confiança

Semelhante ao encontrado em outros trabalhos, observou-se maior frequência de atividade física entre os homens que entre as mulheres, embora não tenha sido estatisticamente significante, mas não foi observada associação com a escolaridade, provavelmente por se tratar de população idosa, grupo em que há mais pessoas de baixa escolaridade, em especial mulheres ${ }^{14}$, mesmo que com condição social privilegiada $^{9,14,18}$. Não se observou, tampouco, associação com a idade, diferentemente do que foi relatado por Siqueira et al. ${ }^{22}$, que identificaram um gradiente positivo de sedentarismo com a idade para os maiores de 65 anos.

Embora sem significância estatística, observou-se uma relação inversa da prática de atividade física com o grau de dependência e com o fato de o idoso apresentar comorbidades, principalmente com três ou mais agravos para os quais o sedentarismo é fator de risco. Mais da metade dos residentes apresentava certo grau de dependência e diversas comorbidades, o que deve mobilizar o profissional no sentido da necessidade de indicação adequada da prática de atividade física para que esses idosos não desenvolvam incapacidades ou piora da sua condição de saúde. No entanto, deve-se ressaltar que o percentual de moderada dependência foi baixo, chamando a atenção para o grau de institucionalização de idosos com leve dependência, inclusive independentes ${ }^{16}$.
Concordando com Silveira et al. ${ }^{22}$, a autoavaliação do estado de saúde mostrou-se positivamente associada à prática de atividade física. Tais resultados podem apontar para uma interdependência entre a prática de exercício e a saúde, indicando que menor prática está associada a piores condições de saúde, e que estas, por sua vez, podem promover a redução na prática de exercício físico ${ }^{22}$. Outra face da autoavaliação pode estar relacionada com a autoestima e com a maneira como o idoso se motiva frente à vida. Tais achados reforçam a necessidade de estimular o mais precocemente possível a prática de atividade física, utilizando estratégias direcionadas a essa população. No entanto, deve-se ressaltar que há benefícios gerados pela prática de atividade física mesmo se for observada tardiamente ${ }^{17,25}$.

Uma das hipóteses que podem explicar o maior percentual de atividade física para os idosos da CGABEG que o observado em outros trabalhos é a inclusão do programa de exercício e caminhada na instituição, atuando como um facilitador para a adesão inicial. Essa hipótese é reforçada pela observação de que um quarto dos indivíduos analisados iniciou a prática de atividade física após ingressarem na instituição, além de não ter sido observada diferença com a idade, conforme relatado por outros autores ${ }^{16}$. Adicionalmente, os responsáveis por esse programa procuram estimular os residentes à participação. 
Os idosos que participam tendem a motivar outros idosos, auxiliando na captação.

Os programas de terapia ocupacional devem ser outro ponto a ser destacado na explicação de maior frequência de atividade física na Casa, uma vez que os idosos, no convívio diário, motivados pela programação, estimulam outros residentes à participação ${ }^{2,9}$. Uma das estratégias mais importantes para o início e a manutenção de prática de exercício é o suporte social dado ao indivíduo. Redes sociais e encorajamento têm-se mostrado importantes influências para o início e a mudança para comportamentos saudáveis ${ }^{1}$.

Segundo Thurston e Green ${ }^{19}$, em discussão sobre a adesão ao exercício na vida madura, chamam a atenção para o fato de que os indivíduos costumam reduzir a atividade no momento de lazer com a família, tendendo a adotar programas domiciliares e, com isso, maior isolamento e menos dispêndio de energia, com consequente redução da condição de saúde desses idosos ${ }^{18}$. Outra questão levantada é que quanto mais cedo o exercício é incorporado à rotina de um indivíduo, maior é a chance de ele manter uma vida ativa em idades mais avançadas $^{19}$. Na CGABEG, os homens são militares reformados e possuíam história de atividade física diária, o que deve explicar a frequência relativamente elevada de prática de exercícios entre os idosos. Destaca-se, ainda, que a maioria que realizava exercício o fazia por tempo suficiente para manter o benefício à saúde.

Uma vez que o sedentarismo é mais prevalente na população idosa e essa população possui perfil de saúde e de comportamento diferenciado em relação à população adulta em geral, é importante o desenho de programas especiais para esse grupo populacional. A prescrição do exercício adequado a essa população e a modificação do comportamento são quesitos importantes a serem considerados. A prescrição do exercício deve contemplar intensidade, frequência, duração, modalidade da atividade física. A escolha de cada componente deve levar em consideração a condição física de cada indivíduo e se o idoso tem história de inatividade de longa data. Existe uma lacuna no conhecimento científico sobre os limites de segurança e sobre o nível de benefício alcançado em função da intensidade de exercício para a população idosa.

A modalidade de exercício também deve ser levada em consideração na prescrição ao paciente ou em programas de atividade física para essa população. A informação de 5 grandes levantamentos realizados na população do sexo masculino maior de 65 anos da Inglaterra, dos Estados Unidos e da Holanda mostrou que a caminhada foi uma das atividades mais realizadas ${ }^{17}$. Dos idosos do CGABEG, aproximadamente todos que realizavam exercício faziam caminhada, embora outras modalidades estivessem disponíveis na instituição.

$\mathrm{O}$ encorajamento através das recomendações médicas melhora a adesão à atividade física ${ }^{24,19}$. É senso comum que a prescrição de atividade física auxilia na manutenção dessa prática $^{19}$. Siqueira et al. ${ }^{22}$ discutem que, muito embora se conheçam os benefícios da prática de atividade física, ainda é baixa a prescrição de exercícios físicos por profissionais de unidades básicas de saúde e unidades do Programa de Saúde da Família. Os profissionais devem ser estimulados a prescreverem exercícios físicos baseados em evidência científica, orientando os limites dessa prescrição.

Uma limitação importante da pesquisa foi não utilizar instrumentos validados para avaliação funcional e contar apenas com a informação do idoso sobre as características de sua prática de atividade física atual e pregressa. A avaliação funcional baseou-se na informação no momento da admissão e não no da pesquisa. No entanto, o tempo de residência não foi elevado e supomos que pouca modificação tenha ocorrido no nível de dependência durante o período.

\section{REFERÊNCIAS}

1. Matsudo SM, Matsudo VKR, Neto TLB. Atividade física e envelhecimento: aspectos epidemiológicos. Rev Bras Med Esporte. 2001;7(1):2-13.

2. Berlezi EM, Rosa PV, Souza ACA, Schneider RH. Comparação antropométrica e do nível de aptidão física de mulheres acima de 60 anos praticantes de atividade física regular e não praticantes. Rev Bras Geriatr Gerontol. 2006;9(3):49-66.

3. Mahecha MS, Rodrigues MVK, Leite BNT. Atividade física e envelhecimento: aspectos epidemiológicos. Rev Bras Med Esporte. 2001;7(1):2-13. Disponível em: http://www.scielo.br/scielo.php?script=sci_ arttext\&pid=S1517-86922001000100002\&lng=en
4. Blair SN, Cheng Y, Holder JS. Is physical activity or physical fitness more important in defining health benefits? Med Sci Sports Exerc. 2001;33(6):379-99.

5. VuorI IM. Health benefits of physical activity with special reference to interaction with diet. Public Health Nutr. 2001;4(2B):517-28.

6. Jacoby E, Bull F, Neiman A. Cambios acelerados del estilo de vida obligan a fomentar la actividad física como prioridad en la región de las Américas. Rev Panam Salud Pública. 2003;4(4):223-5.

7. Bernstein MS, Morabia A, Sloutskis D. Definition and prevalence of sedentarism in an urban population. Am J Public Health. 1999;89(6):862-7. 
8. Gomes VB, Siqueira KS, Sichieri R. Atividade física em uma amostra probabilística da população do Município do Rio de Janeiro. Cad Saúde Pública. 2001;17(4):969-76.

9. Agita São Paulo, 2005 Dia do Idoso Ativo: Caminhada para um envelhecimento saudável. Available from: http://www.agitasp.com.br/idoso2.asp

10. Pitanga FJG, Lessa I. Prevalência e fatores associados ao sedentarismo no lazer em adultos. Cad Saúde Pública. 2005;21(3):870-7.

11. Florindo AA, Latorre MRDO, Tanaka T, Jaime PC, Zerbini CAF. Fatores associados à prática de exercício físico em homens voluntários adultos e idosos residentes na Grande São Paulo. Rev Bras Epidemiol. 2001;4(2):105-13.

12. Thurston M, Green K. Adherence to exercise in later: how can exercise on prescription programmes be made more effective? Health Promot Int. 2008;19(3):379-86.

13. Ferreira MT, Matsudo VKR, Braggion GF. Efeitos de um programa de orientação de atividade física e nutricional sobre a ingestão alimentar e composição corporal de mulheres fisicamente ativas de 50 a 72 anos de idade. Rev Bras Ciênc Mov. 2003;11(1):35-40.
14. Kleinbaum DG, Klein M. Logistic regression: a self learning text. 2nd ed. Springer-Verlag New York; 2002.

15. Siqueira, F V, Facchini La, Piccini RX. Atividade física em adultos e idosos residentes em áreas de abrangências de unidades básicas de saúde de municípios das regiões Sul e Nordeste do Brasil. Cad Saúde Pública. 2008;24(1):39-54.

16. Chaimowicz F. A saúde dos idosos brasileiros às vésperas do século XXI: problemas, projeções e alternativas. Rev Saúde Pública. 1997;31(2):184-200.

17. Chaimowicz F, Greco DB. Dinâmica da institucionalização de idosos em Belo Horizonte, Brasil. Rev Saúde Pública. 1999;33(5):454-60.

18. Song SH. Physical activity in the elderly: it is never too late! Q J Med. 2008;101:743-44.

Recebido em: 19/03/2012

Aprovado em: 19/06/2012 